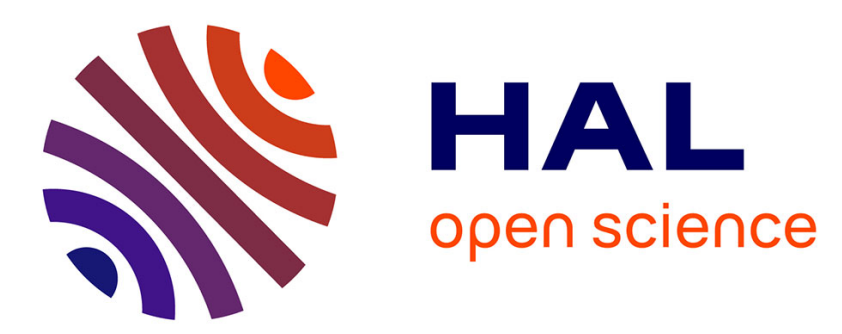

\title{
Experimental investigation of fatigue crack growth behavior of GH2036 under combined high and low cycle fatigue
}

Dianyin Hu, Fanchao Meng, Huawei Liu, Jun Song, Rongqiao Wang

\section{To cite this version:}

Dianyin Hu, Fanchao Meng, Huawei Liu, Jun Song, Rongqiao Wang. Experimental investigation of fatigue crack growth behavior of GH2036 under combined high and low cycle fatigue. International Journal of Fatigue, 2016, 85, pp.1-10. 10.1016/j.ijfatigue.2015.10.027 . hal-01321519

\author{
HAL Id: hal-01321519 \\ https://hal.science/hal-01321519
}

Submitted on 25 May 2016

HAL is a multi-disciplinary open access archive for the deposit and dissemination of scientific research documents, whether they are published or not. The documents may come from teaching and research institutions in France or abroad, or from public or private research centers.
L'archive ouverte pluridisciplinaire HAL, est destinée au dépôt et à la diffusion de documents scientifiques de niveau recherche, publiés ou non, émanant des établissements d'enseignement et de recherche français ou étrangers, des laboratoires publics ou privés.

\section{다(1)(2)}

Distributed under a Creative Commons Attribution - ShareAlikel 4.0 International 


\title{
Experimental investigation of fatigue crack growth behavior of GH2036 under combined high and low cycle fatigue
}

\author{
Dianyin $\mathrm{Hu}^{\mathrm{a}, \mathrm{b}}$, Fanchao Meng ${ }^{\mathrm{c}}$, Huawei Liu ${ }^{\mathrm{a}}$, Jun Song ${ }^{\mathrm{c}}$, Rongqiao Wang ${ }^{\mathrm{a}, \mathrm{b}, *}$

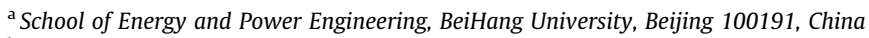 \\ ${ }^{\mathrm{b}}$ Collaborative Innovation Center of Advanced Aero-Engine, Beijing 100191, China \\ ${ }^{\mathrm{c}}$ Mining and Materials Engineering, McGill University, Montreal, QC H3A 0C5, Canada
}

\begin{abstract}
Fatigue crack growth rates have been experimentally determined for the superalloy GH2036 (in Chinese series) at an elevated temperature of $550{ }^{\circ} \mathrm{C}$ under pure low cycle fatigue (LCF) and combined high and low cycle fatigue (CCF) loading conditions by establishing a CCF test rig and using cornernotched spec-imens. These studies reveal decelerated crack growth rates under CCF loading compared to pure LCF load-ing, and crack propagation accelerates as the dwell time prolongs. Then the mechanism of fatigue crack growth at different loadings has been discussed by using scanning electron microscope (SEM) analyses of the fracture surface.
\end{abstract}

\section{Introduction}

Turbine discs are important load-bearing components in modern aero-engines, whose structural integrity is critical to engine performance. Generally a turbine disc is connected with turbine blades by means of a fir-tree design [1], i.e., a typical multiple load path structure, to allow for different rates of expansion between the disc and blade while still holding the blade firmly against centrifugal loads. During operation, turbine discs experience significant tensile stresses due to centrifugal loading and thermal loading. In addition, they may also experience aerodynamically induced small-amplitude and high-frequency vibrations. The centrifugal and thermal stresses can cause turbine discs to fail by low-cycle fatigue (LCF), and meanwhile the aerodynamically induced vibration stresses can lead to high-cycle fatigue (HCF) failures [2-4]. This combined loading on the gas turbine disc posts serious reliability concerns and thus requires careful investigation.

Damage-tolerant design approach has been successfully used to reduce fatigue failures and to extend lifetimes of critical components [5]. In this approach, the predicted lifetime is calculated by integrating the $d a / d N$ versus $\Delta K$ curve. This approach is also shown to work well under combined high and low cycle fatigue (CCF) loading [6]. Fatigue crack propagation behavior due to the combined action of LCF and HCF is therefore seen to be of broad interest [4]. Several studies on titanium alloys and steels stated that the HCF cycles lead to accelerated crack growth rates in comparison to pure LCF loading as soon as the total stress intensity $\Delta K$ range overcomes a certain threshold $\Delta K_{\text {onset }}[4,6-10]$. Meanwhile, the influence of the number of HCF cycles per LCF cycle on fatigue crack growth was investigated systematically. While the conclusion that the superimposed HCF loadings accelerate the growth of fatigue crack and thus decrease the fatigue life has been well drawn in literature, several studies on some certain superalloys, which were tested under CCF loading, showed that HCF cycles with small amplitudes increase the number of cycles to failure instead [11-14]. The available literature data in $[11,13]$ indicated that the superposition of high frequency low amplitude vibrations on LCF loading may be both negative and positive as regards the number of LCF cycles to failure. It was dependent on the ratio of the amplitudes of HCF to LCF loading. When the ratio was large enough, shorter lifetime was observed as expected. However, for small ratio the effect of HCF amplitudes resulted in an increase of LCF lifetime.

The material of interest in the present study is the precipitation hardened iron-based superalloy GH2036, which is optimized for good mechanical properties at high temperatures, and good resistance to high temperature corrosion and oxidation [15]. It has been widely used for rotating structural components, including engine 

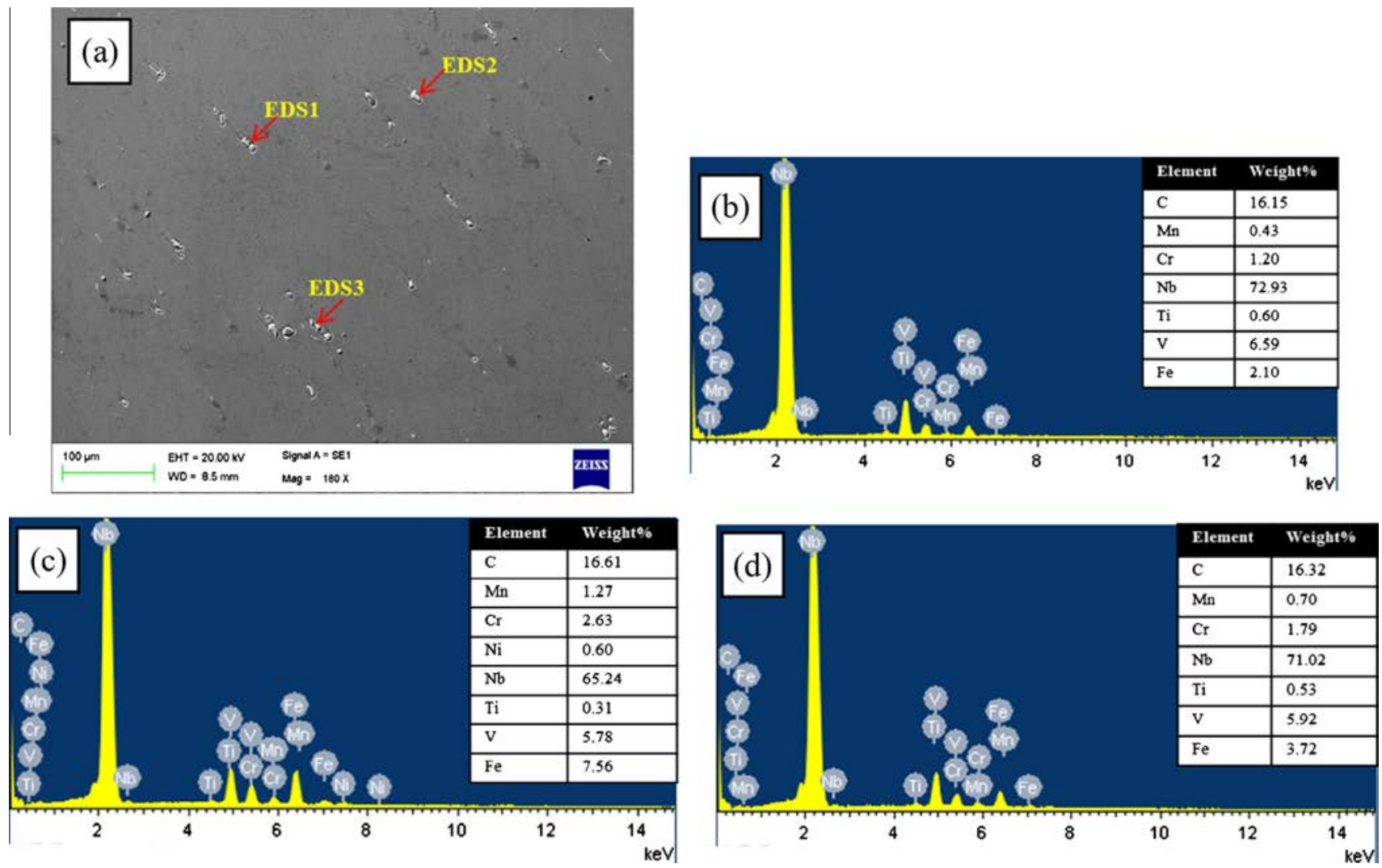

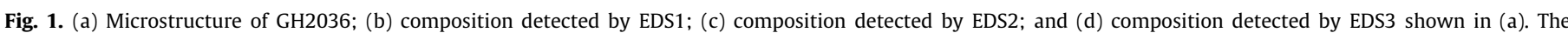
quantitative results corresponding to the EDS spectrum are inserted in (b)-(d) respectively.
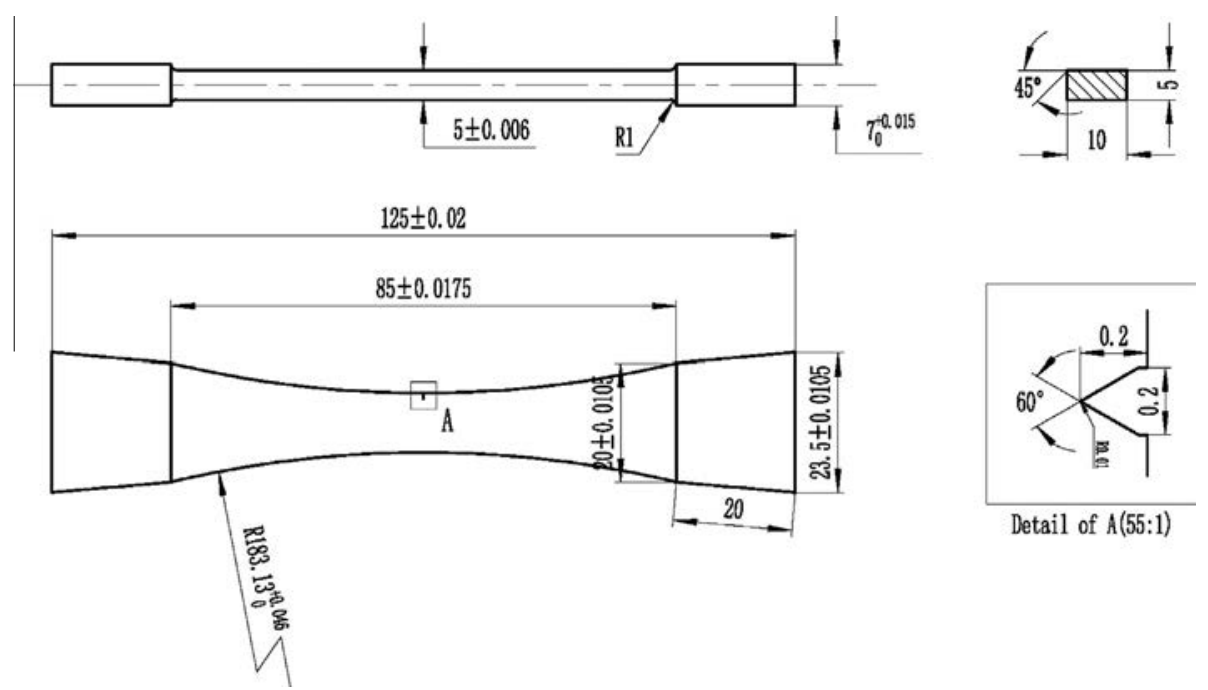

Fig. 2. Geometries of plate specimens for fatigue crack growth test (unit: mm).

Table 1

Chemical content of GH2036 (wt\%).

\begin{tabular}{llllllllll}
\hline $\mathrm{Fe}$ & $\mathrm{C}$ & $\mathrm{Si}$ & $\mathrm{Mn}$ & $\mathrm{Cr}$ & $\mathrm{Ni}$ & $\mathrm{Mo}$ & $\mathrm{Nb}$ & $\mathrm{Ti}$ & $\mathrm{V}$ \\
\hline Balance & 0.37 & 0.5 & 8.5 & 12.0 & 8.0 & 1.25 & 0.35 & $\leqslant 0.12$ & 1.4 \\
\hline
\end{tabular}

turbine discs and blades constantly subjected to large stress ratios and high temperatures [16-18]. In this paper, the fatigue crack propagation behaviors of $\mathrm{GH} 2036$ under $\mathrm{CCF}$ loading at $550{ }^{\circ} \mathrm{C}$ corresponding to the turbine disc's working temperature are studied. Targeted experiments which achieved a synchronous treatment of the high cycle and low cycle loading are designed and performed to investigate the mechanism underlying the fatigue crack propagation of GH2036 under CCF loading. Three specimens are conducted under pure LCF loading, and the other three specimens were tested under CCF loading. The influence of the $\mathrm{HCF} / \mathrm{LCF}$ cycle ratio (i.e. the number of HCF cycles per LCF cycle) on fatigue crack growth was investigated, two group tests of different number of HCF cycles are conducted which reveals the influence of the amount of HCF cycles. The fatigue life of GH2036 


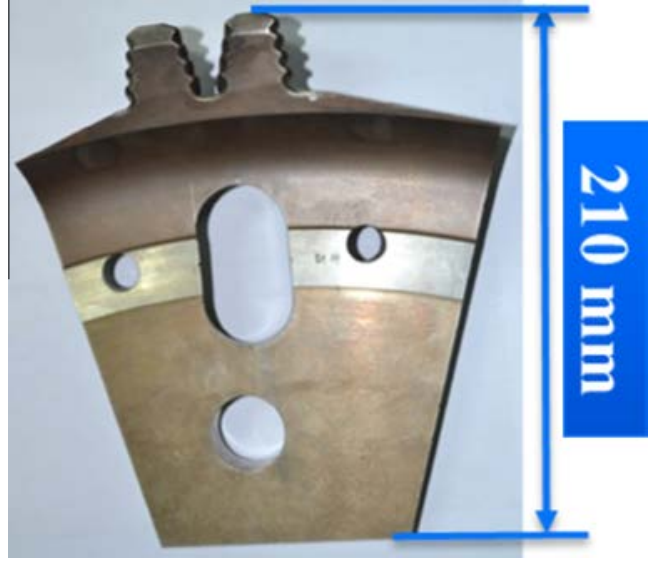

Fig. 3. The sector of actual turbine disc.

under both the LCF and CCF loadings at $550{ }^{\circ} \mathrm{C}$ in air is experimentally determined. In the end, the mechanisms of fatigue crack growth of GH2036 superalloy under different loadings are discussed and our results are summarized.

\section{Experimental details}

\subsection{Material}

The experiments were performed on turbine discs made of GH2036 superalloy, the chemical composition of which is listed in Table 1. Fig. 1(a) shows the microstructure of the material revealed by scanning electron microscope (SEM). An energy dispersive spectroscopy (EDS) analysis of the microstructure indicates that GH2036 superalloy is strengthened by a random distribution of $\mathrm{NbC}$ and VC precipitates (Fig. 1(b)-(d)).

\subsection{Specimen geometry}

The specimen geometry for fatigue testing is shown in Fig. 2. The corner-notched specimen has a rectangular cross section with a thickness and width of $5 \mathrm{~mm}$ and $10 \mathrm{~mm}$ respectively. The specimen orientation, as defined by ASTM notation, was longitudinalshort transverse, which corresponds to the growth of the corner through the length of the fir-tree mortise in response to centrifugally induced stresses. Each specimen was pre-cracked at room temperature, at a stress ratio of 0.01 and a frequency of $5.0 \mathrm{~Hz}$ in order to generate a near quarter-circular crack with a radius of $0.2 \mathrm{~mm}$. To minimize the effects of pre-cracking at room temperature on the test at $550{ }^{\circ} \mathrm{C}$, we ensure that the plastic zone sizes at the end of the precracking stage were the same as the start of the crack growth rate testing [7]. The size and shape of the developed crack with this specimen are more representative of turbine disc's operation conditions than those reproducted in a compact tension or bend specimen $[6,8,19-20]$. All the test pieces were cut from a forged turbine disc of GH2036 given in Fig. 3. The turbine disc is solution heat treated at a temperature of $1120^{\circ} \mathrm{C}$, and held for $1 \mathrm{~h}$, followed by an air cool. Following the solution heat treatment, the disc undergoes a stabilization heat treatment at $870{ }^{\circ} \mathrm{C}$ for $1 \mathrm{~h}$, followed by an air cool. Finally, the disc is heated to $650^{\circ} \mathrm{C}$ for $24 \mathrm{~h}$, followed by an air cool.

\subsection{Procedures for fatigue crack growth test}

Although single actuator test machines could generate the required waveforms, their ability to do so is severely limited. In this regard, we built a new test rig, illustrated in Fig. 4(a), in order to achieve a rational and noninterfering CCF loading [21]. In this test rig, particular fixture has been designed to avoid the interactions between the control systems for the two loadings by employing a load train assembly with three movable joints in Fig. 4(b) to allow the specimen to bend. A pair of wedge-shape clamping

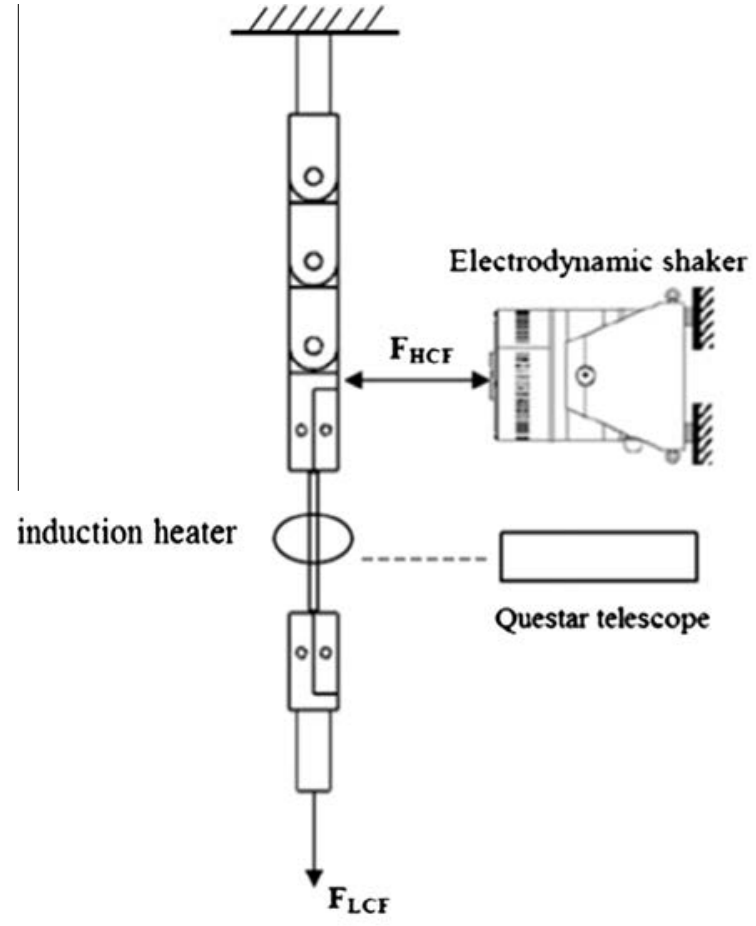

(a)
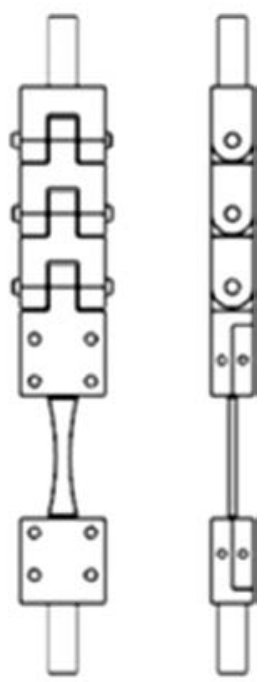

(b)

Fig. 4. (a) A sketch of the new CCF loading method with actuators, long-focus microscope and electromagnetic induction heater (b) load train assembly. 


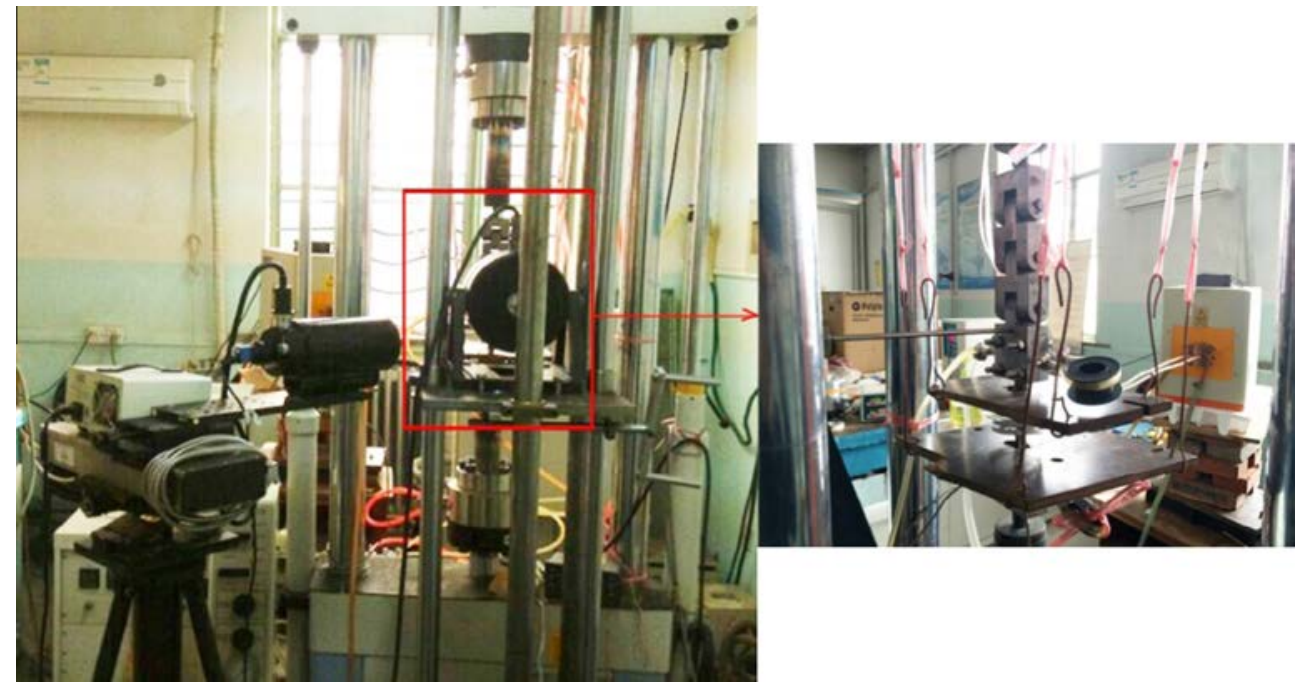

Fig. 5. CCF test was being conducted.

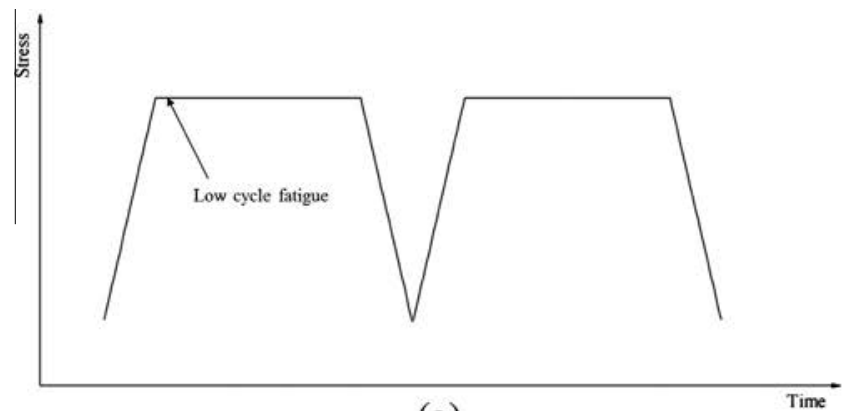

(a)

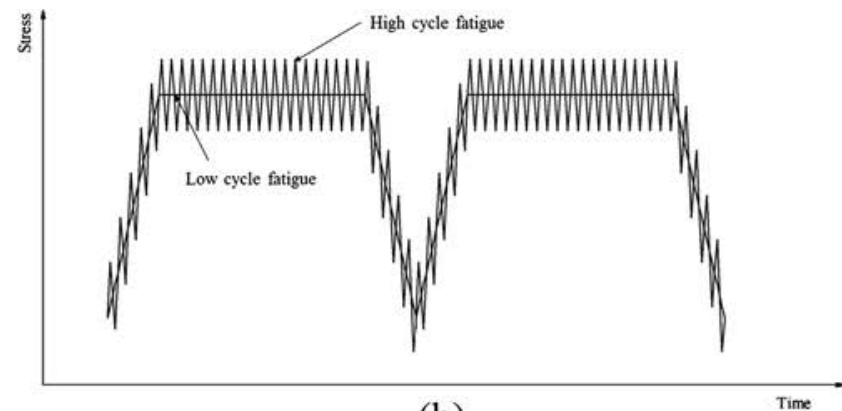

(b)

Fig. 6. Diagrammatic representation of (a) loading waveform for pure LCF tests, and (b) loading waveform for CCF tests.

Table 2

Overview over all LCF and CCF tests.

\begin{tabular}{llll}
\hline $\begin{array}{l}\text { Loading } \\
\text { conditions }\end{array}$ & $\begin{array}{l}\text { Cycle ratio of minor cycle per } \\
\text { major cycle, } n\end{array}$ & $\begin{array}{l}\text { Dwell } \\
\text { time }(\mathrm{s})\end{array}$ & $\begin{array}{l}\text { Specimen } \\
\text { number }\end{array}$ \\
\hline Pure LCF & $/$ & 9 & $\# 1, \# 2$ \\
CCF & $1000: 1$ & 9 & $\# 4, \# 5$ \\
Pure LCF & $/$ & 108 & $\# 3$ \\
CCF & $10,000: 1$ & 108 & $\# 6$ \\
\hline
\end{tabular}

device was used to hold the specimen when LCF loading was applied by using a servo-hydraulic actuator. In addition, four smaller bolts shown in Fig. 4(b) were employed to maximize the damping of the load train by increasing the surface contact stiffness between the clamping device and specimen. The HCF loading with a $200 \mathrm{~N}$ electrodynamic shaker to excite up to $5000 \mathrm{~Hz}$ and an $8 \mathrm{~mm}$ amplitude was transferred to the clamping device through the vibration exciting rod. It is worth pointing out that with the structure of three movable joints, the interference between HCF loading and LCF loading can be eliminated and the loading efficiency of HCF is greatly improved because of the decrease of friction. Thus, a rational and noninterfering CCF loading is achieved.

The specimen was heated by a high-frequency induction heater. The temperature of the specimen was monitored by a $K$-type thermocouple placed in the center of the gauge length. Test temperature was $550{ }^{\circ} \mathrm{C}$, equal to the maximum continuous working temperature of the turbine disc. All the experiments were run in

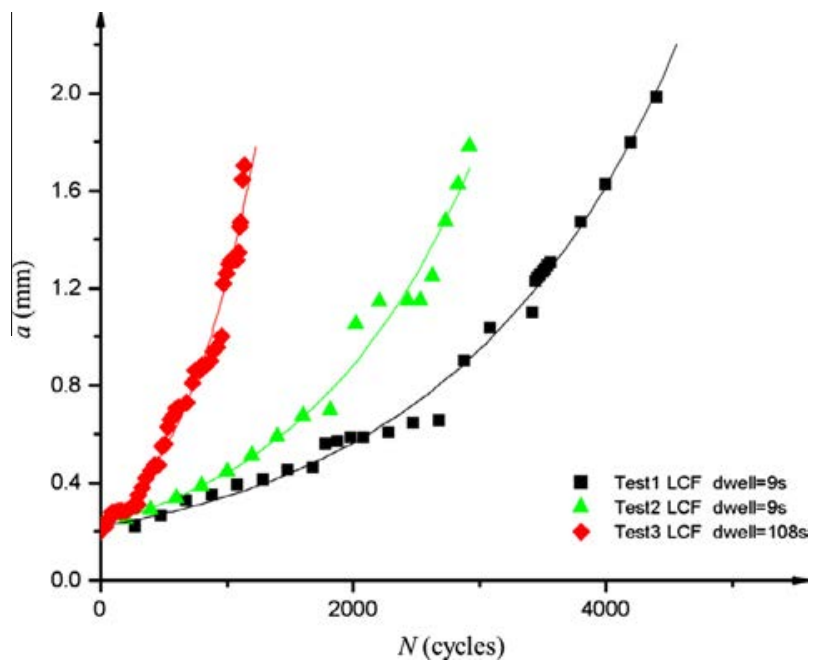

Fig. 7. Crack length evolution under pure LCF loading at $9 \mathrm{~s}$ and $108 \mathrm{~s}$ dwell time The temperature is $550{ }^{\circ} \mathrm{C}$. The solid lines represent the exponential fitting of data.

laboratory air. There were also validation and control measures regarding to LCF and HCF loading. For LCF loading, four strain gauges were stuck on the key section. For the HCF loading, test results manifested that the vibration stress of the key section had a linear relation with vibration amplitude of the excitation 


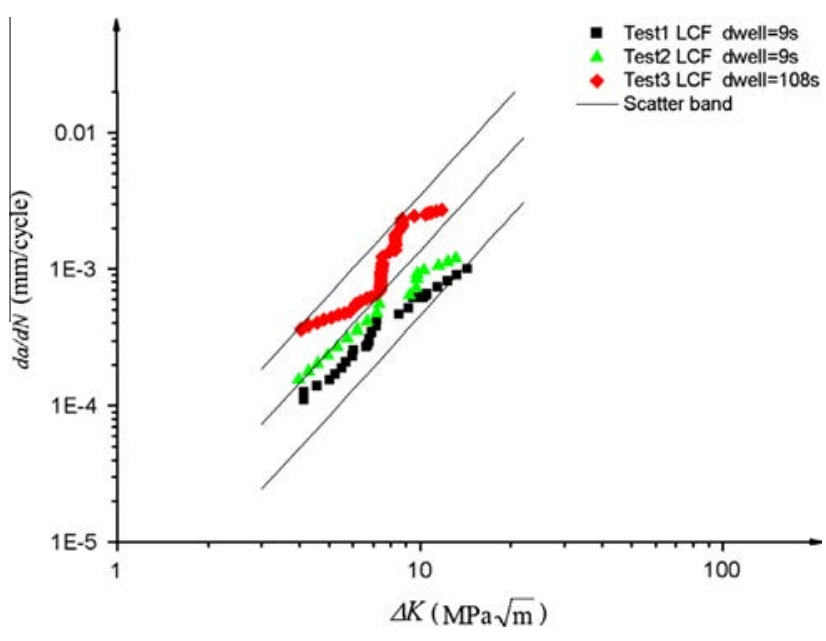

Fig. 8. Fatigue crack growth rates under pure LCF loading at $9 \mathrm{~s}$ and $108 \mathrm{~s}$ dwell time. Test data boundary lines (solid black lines) are included. The temperature is $550{ }^{\circ} \mathrm{C}$.

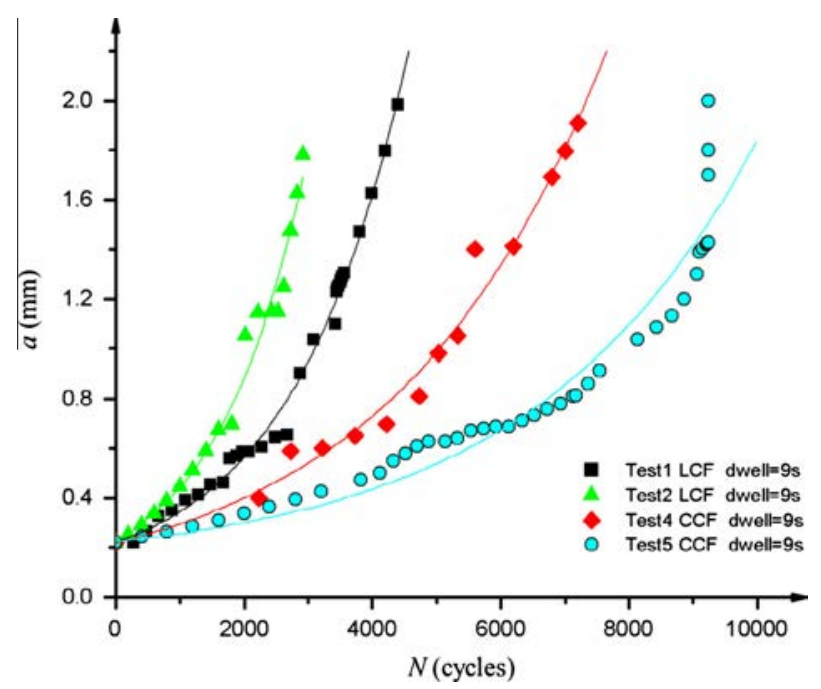

Fig. 9. Evolution of crack length under CCF loading at a cycle ratio of 1000:1 and LCF loading conditions at a dwell time of $9 \mathrm{~s}$. The temperature is $550{ }^{\circ} \mathrm{C}$. The solid lines represent the exponential fitting of data.

point [22]. Accordingly, the excitation point was monitored and controlled by a vibrometer in order to insure the accuracy of the HCF loading.

Crack lengths were measured by using a Questar telescope with a $2 \mu \mathrm{m}$ resolution, which was verified to have a high degree of confidence [23]. Stress intensity factors were calculated by the following relationship developed by Pickard [24] for the free surface position of a quarter-circular crack growing under tension shown in Eq. (1):

$K=M_{\mathrm{G}} \cdot M_{\mathrm{B}} \cdot M s \cdot F(\sigma) \cdot \Phi \cdot(\pi c)^{1 / 2}$

where $M_{\mathrm{G}}$ is the comprehensive correction coefficient and is related to the type and position of crack, $M_{\mathrm{B}}$ is back-face correction factor, and $M_{\mathrm{S}}$ is side-face correction factor. $F(\sigma)$ is stress function representing the effect of the complicated stress field on the stress intensity factor. $\Phi$ is correction factor of ellipse, and is equal to $2 / \pi$ in this paper. $c$ represents the distance between the crack center and the crack front.

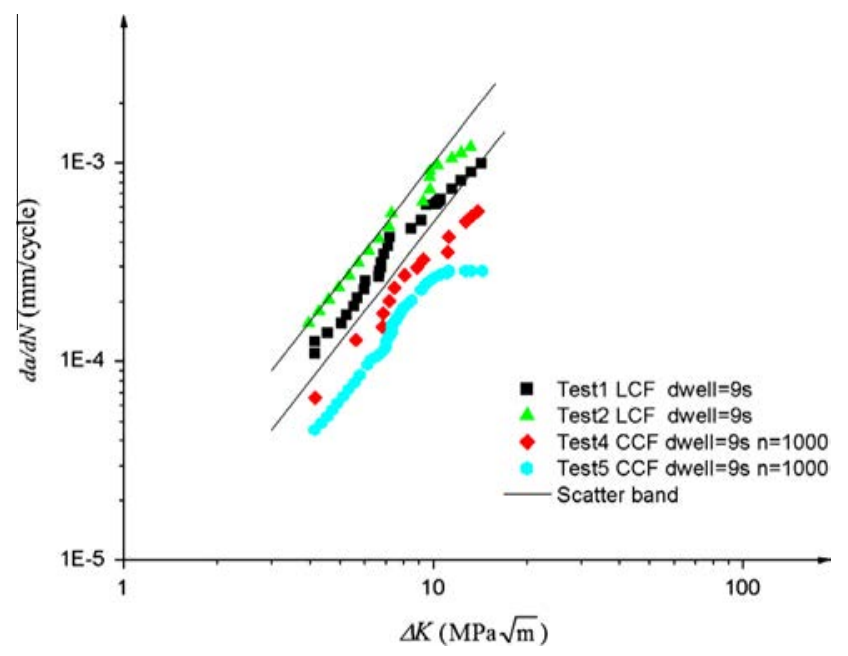

Fig. 10. Evolution of fatigue crack growth rate under CCF loading at a cycle ratio of 1000:1 and under LCF loading at a dwell time of $9 \mathrm{~s}$. Crack growth data boundary lines for pure LCF loading are included (solid black lines). The temperature is $550^{\circ} \mathrm{C}$.

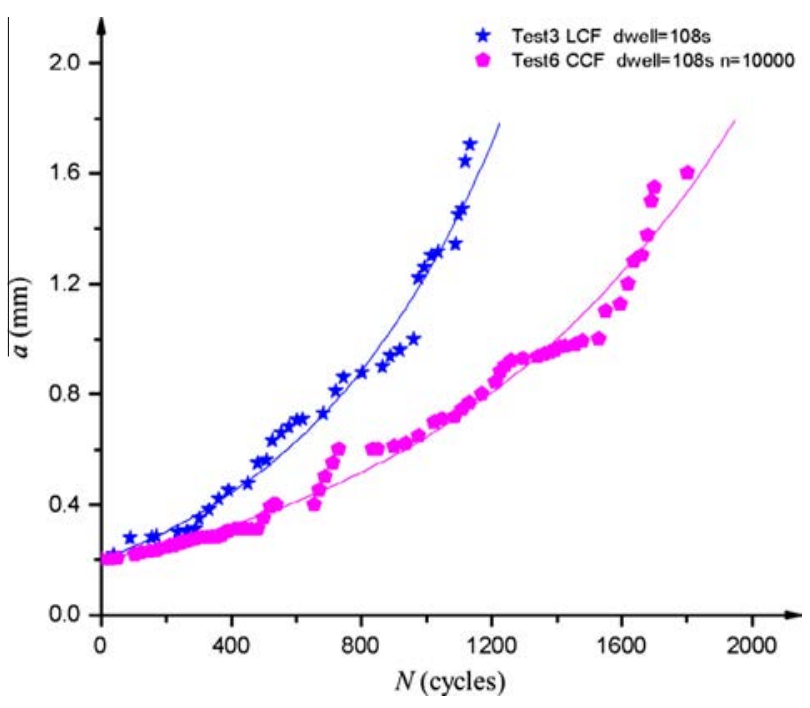

Fig. 11. Evolution of crack length under CCF loading at a cycle ratio of 10,000:1 and LCF loading condition at a dwell time of $108 \mathrm{~s}$. The temperature is $550^{\circ} \mathrm{C}$. The solid lines represent the exponential fitting of data.

Crack growth rate $d a / d N$ was determined by the 3-point secant method of analysis. Fig. 5 shows the CCF experiment being conducted.

The waveform of the LCF loading was simplified and represented by a trapezoidal stress wave with $1 \mathrm{~s}$ rise and fall times with a stress ratio $\left(R_{\mathrm{LCF}}\right)$ of 0.1 , as shown in Fig. 6(a). In the test programme two LCF loading blocks tests were undertaken using different dwell time in order to assess the importance of time dependent fatigue crack growth with $9 \mathrm{~s}$ and $108 \mathrm{~s}$ dwells at the maximum load, which results in LCF loading frequencies of 0.091 and $0.0091 \mathrm{~Hz}$, respectively. A loading frequency of $91 \mathrm{~Hz}$ was superimposed on the LCF cycle dwell period at the maximum load, the conjoint action of which is referred to as CCF, as illustrated in Fig. 6(b). The value of $91 \mathrm{~Hz}$ was the resonant frequency of the test facility. The stress ratio of HCF cycle $\left(R_{\mathrm{HCF}}\right)$ was 0.95 . The stress amplitude ratio of LCF to HCF cycles, $Q$ is defined as:

$Q=\frac{\left(1+R_{\mathrm{HCF}}\right)\left(1-R_{\mathrm{LCF}}\right)}{2\left(1-R_{\mathrm{HCF}}\right)}=\frac{(1+0.95)(1-0.1)}{2(1-0.95)}=17.55: 1$ 


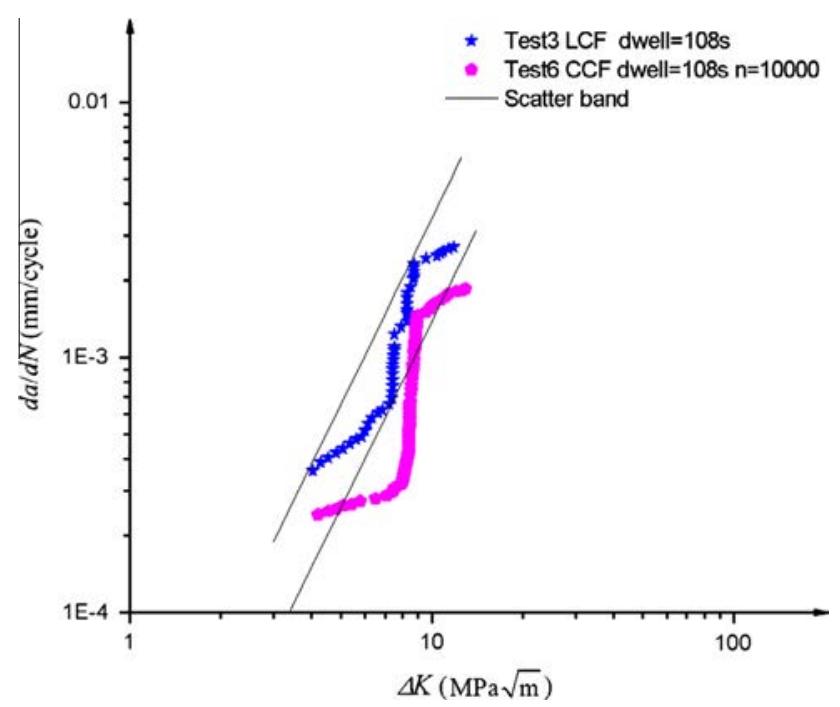

Fig. 12. Fatigue crack growth rates for CCF loading at cycle ratio of $10,000: 1$ and for LCF loading at a $108 \mathrm{~s}$ dwell time. Crack growth data boundary lines (solid black lines) for LCF loading are included. The temperature is $550{ }^{\circ} \mathrm{C}$.

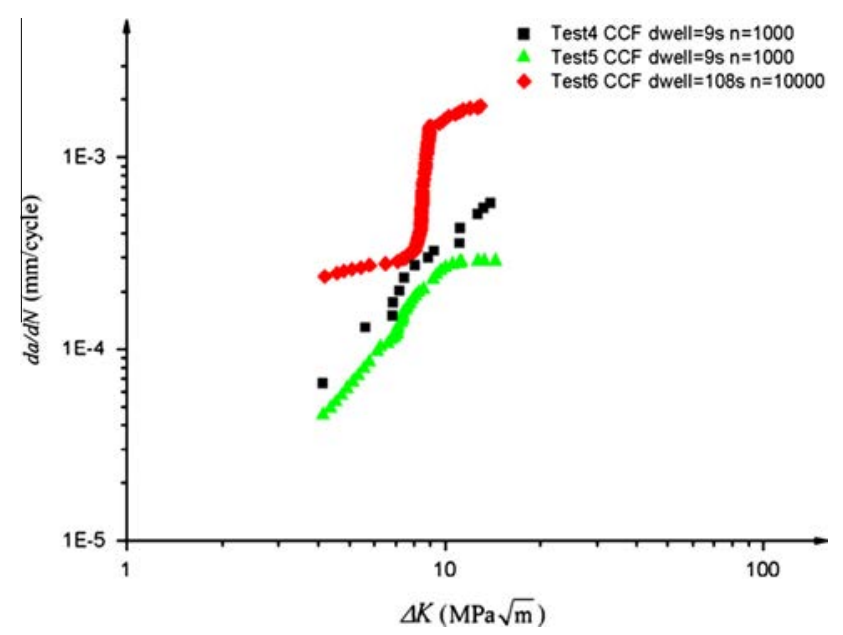

Fig. 13. The comparison of fatigue crack growth rate under CCF tests with the cycle ratio of $1000: 1$ and $10,000: 1$. The temperature is $550{ }^{\circ} \mathrm{C}$.

In the CCF tests, the use of a $9 \mathrm{~s}$ dwell at maximum load resulted in a cycle ratio $(n)$ of 1000:1 HCF cycle for every LCF cycle, whilst the $108 \mathrm{~s}$ dwell at maximum load gave a cycle ratio (n) of 10,000:1. An overview over all LCF and CCF tests is given in Table 2. Traverses were made across each specimen surface, one from the crack origin to the diagonally opposite corner and another along one edge. By correcting the orientation of the microscope and eliminating tilting of the specimen, photographs were taken at approximately $0.5 \mathrm{~mm}$ intervals across the fracture surface. The crack length associated with each photograph was measured using the machine's traversing micro-meters.

\section{Results and discussions}

\subsection{Crack growth rate under pure LCF loading}

The crack length evolution of three specimens under pure LCF loading at the $9 \mathrm{~s}$ and $108 \mathrm{~s}$ dwell time is displayed in Fig. 7. Fig. 8 demonstrates $d a / d N$ obtained from three pure LCF loading

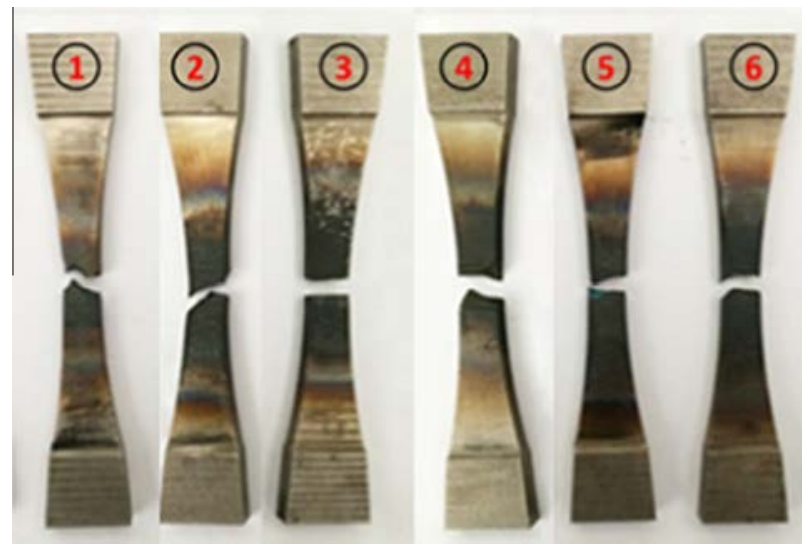

Fig. 14. Photo of tested specimens.

tests with respect to the stress intensity factor range $\Delta K$ defined by Eq. (1) on logarithmic axes. It is shown that crack growth data under LCF loading reveals scatter due to the random distribution in the microstructure of the test material. Thus it is convenient to replace the scatter of data points by the construction of the band, within which all crack growth data falls (see Fig. 8). The slopes of the least squares lines are 2.4 and 2.0, respectively. The effect of increasing dwell time is to markedly increase the fatigue crack growth rates. Consequently, there is time dependent crack growth for $\mathrm{GH} 2036$ at $550{ }^{\circ} \mathrm{C}$. It is observed from Fig. 8 that there is a strong stepwise increase in $d a / d N$ for a long dwell time of $108 \mathrm{~s}$, which is different from that at the shorter dwell time of $9 \mathrm{~s}$.

\subsection{Effects of HCF cycles on crack growth rate of CCF loading}

The evolution of crack length of the four specimens under CCF and LCF loadings at a $9 \mathrm{~s}$ dwell is shown in Fig. 9. The crack growth rate $d a / d N$ data obtained from two CCF tests at a cycle ratio of 1000:1 and the two pure LCF tests are compared in Fig. 10. The $d a / d N$ data falls outside the bottom band of the pure LCF band and as $\Delta K$ increases, crack growth rate $d a / d N$ moves progressively away from the band. The superimposed HCF loadings lead to decreased crack growth rate and increased lifetime in contrast to pure LCF loading, which demonstrates that the superimposed HCF cycles delay the crack growth rate for GH2036 superalloy at $550{ }^{\circ} \mathrm{C}$.

In addition, another two tests under pure LCF loading and CCF loading with a cycle ratio of 10,000:1 and at a dwell time of $108 \mathrm{~s}$ were carried out. The corresponding data are presented in Figs. 11 and 12, also indicating that the crack growth rate is less under CCF loading than that under pure LCF loading. Most crack growth rate data also falls outside the bottom band (see two solid black lines in Fig. 12) of the pure LCF band. Compared to the data in Fig. 10, the fatigue crack growth rate drops slowly because of superimposed HCF cycles. The stepwise increase in $d a / d N$ appears similar to the crack growth rate under pure LCF loading with a long dwell time (see Fig. 12).

\subsection{Effects of cycle ratio on crack growth rate of CCF loading}

Fig. 13 shows the crack growth data using CCF tests with two cycle ratios. In contrast to CCF loading with a 10,000:1 cycle ratio (i.e. 10,000 HCF cycles are superimposed on every LCF cycle at a $108 \mathrm{~s}$ dwell), the crack growth rate decreases at a cycle ratio of 1000:1 (i.e. 1000 HCF cycles are superimposed on every LCF cycle at a $9 \mathrm{~s}$ dwell). Fig. 8 shows that long dwell time significantly 

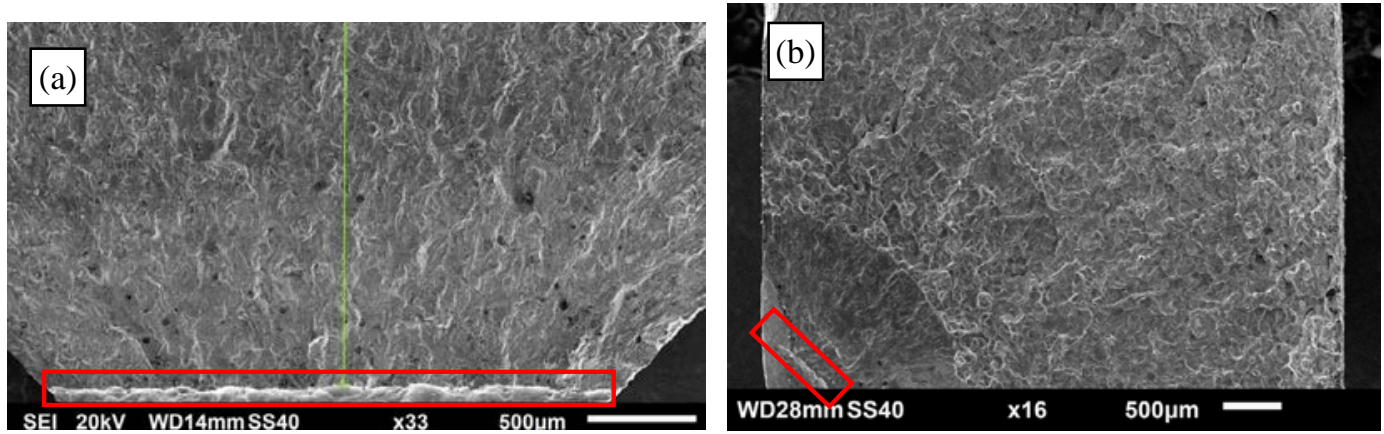

Fig. 15. Fatigue fracture surface. (a) Specimen \#1, and (b) specimen \#3. Red lines show the crack starter notches. (For interpretation of the references to colour in this figure legend, the reader is referred to the web version of this article.)
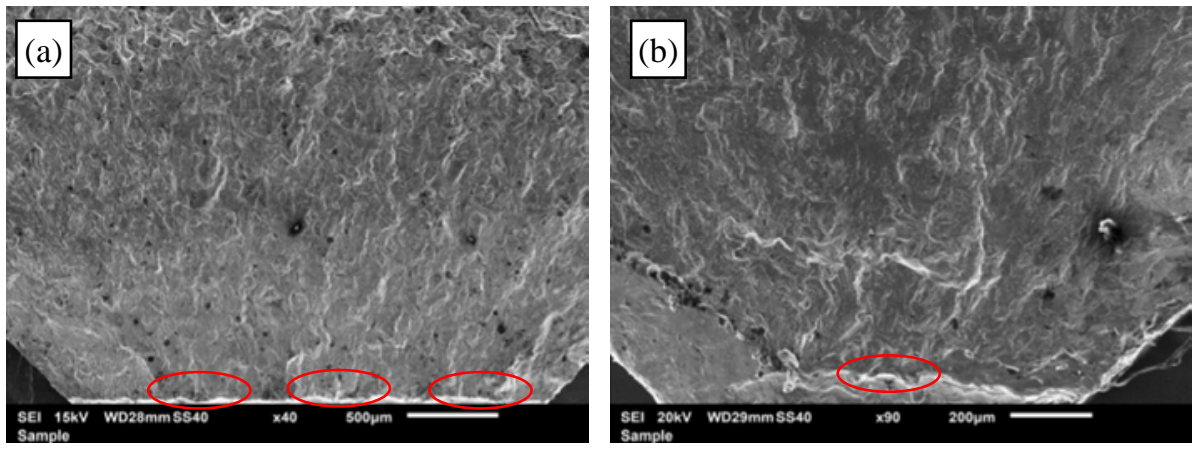

Fig. 16. Fatigue fracture surface at pure LCF loading. (a) Crack initiation of specimen \#1 with a $9 \mathrm{~s} \mathrm{dwell,} \mathrm{and} \mathrm{(b)} \mathrm{specimen} \mathrm{\# 3} \mathrm{with} \mathrm{a} 108 \mathrm{~s}$ dwell. Red ovals indicate the crack initiation zones. (For interpretation of the references to colour in this figure legend, the reader is referred to the web version of this article.)
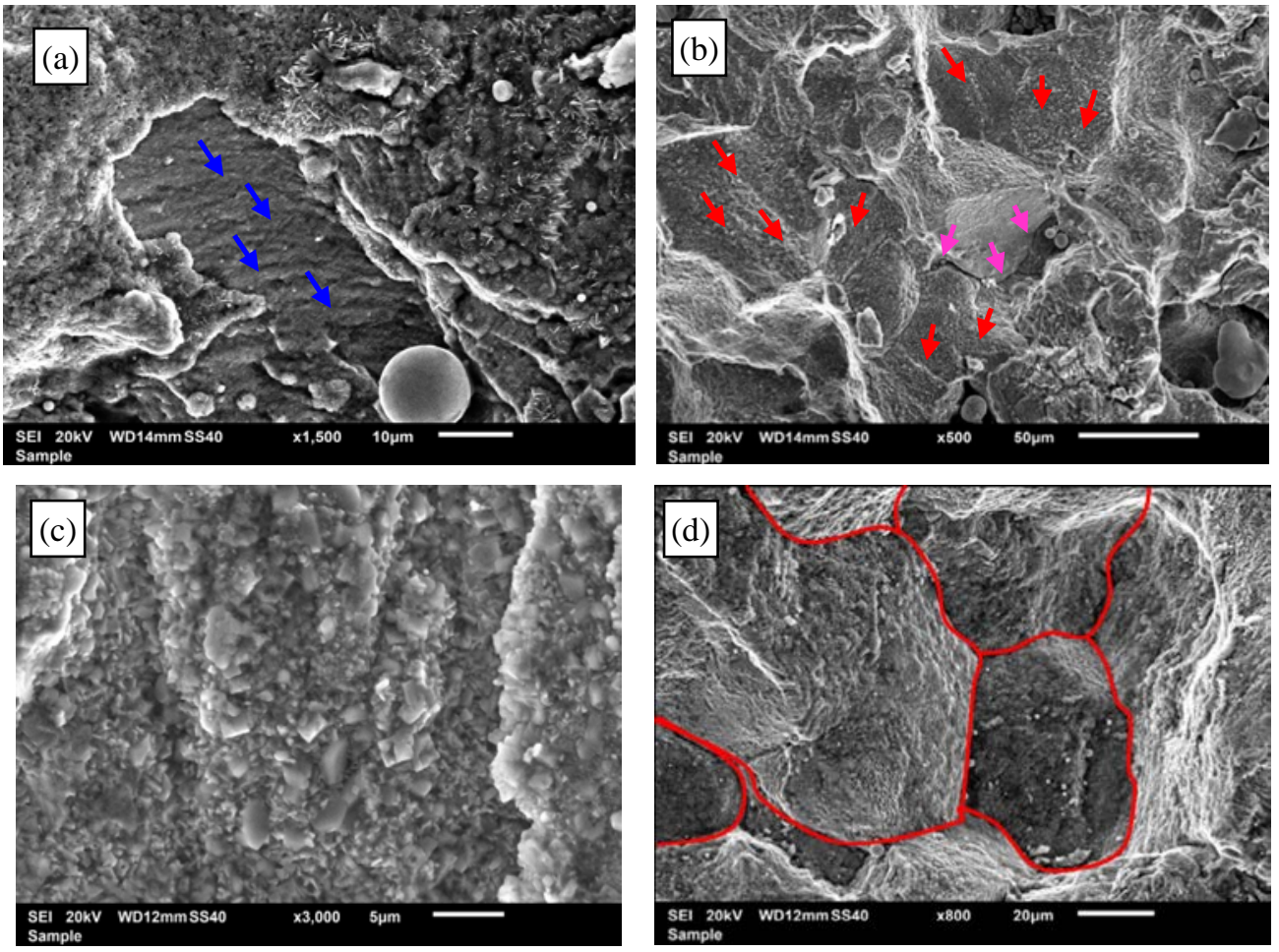

Fig. 17. SEM photos of fracture morphology under pure LCF loading. (a) Specimen \#1 with oxide. Blue arrows indicate fatigue striations, (b) specimen \#1, $\Delta K=8.3 \mathrm{MPa} \sqrt{m}$. Red arrows indicate transgranular fracture. Pink arrows show the intergranular secondary cracks. (c) Specimen \#3 highly oxidized. (d) Specimen \#3, $\Delta K=8.3$ MPa $\sqrt{m}$. Red curves indicate grain boundaries. (For interpretation of the references to colour in this figure legend, the reader is referred to the web version of this article.) 


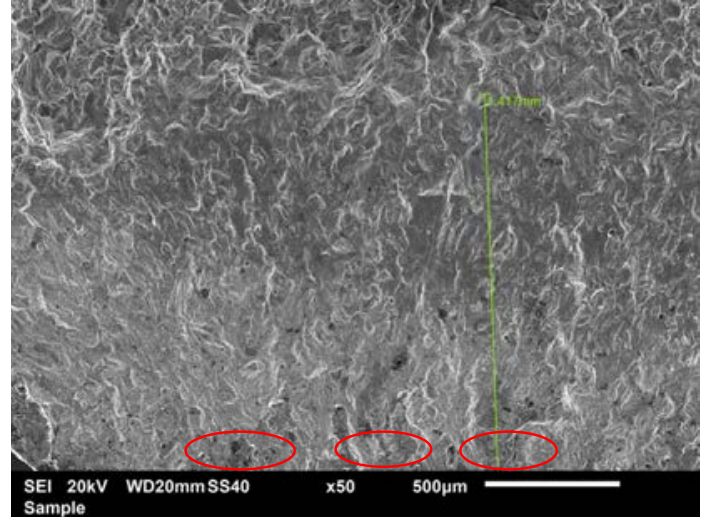

Fig. 18. Crack initiation of specimen \#4 at CCF loading (dwell = $9 \mathrm{~s}, n=1000: 1$ ). Red ovals indicate the crack initiation zones. (For interpretation of the references to colour in this figure legend, the reader is referred to the web version of this article.)

accelerates the fatigue crack growth rate, i.e. the crack under LCF loading with a $108 \mathrm{~s}$ dwell grows faster than with a $9 \mathrm{~s}$ dwell. Also, it is demonstrated that the more HCF cycles are superimposed on LCF loading, the less the crack growth rate decreases. This means that the crack growth rate with CCF loading with a 10,000:1 cycle ratio slightly decreases compared with LCF loading at a $108 \mathrm{~s}$ dwell. Therefore, as the cycle ratio increases, the fatigue crack growth accelerates.

\subsection{Fractography}

A systematic fractographic analysis were performed by SEM on the fractured specimens (see Fig. 14) from the above tests, in which \#1 and \#2 were tested under LCF loading at a 9 s dwell time while
\#3 under LCF loading at a $108 \mathrm{~s}$ dwell time, \#4 and \#5 were tested under CCF loading with a cycle ratio of 1000:1, and \#6 was tested under CCF loading with a cycle ratio of $10,000: 1$. No significant differences were discovered between the fracture surface along the diagonal and along the edge of the specimens, corresponding to the plane strain and plane stress regions respectively. All the specimens initiated and grew the crack from the starter notches as shown in Fig. 15.

\subsubsection{The effect of dwell time under pure LCF loading}

The examination of fracture surfaces by SEM shows that fatigue cracks originate at the surface. Fig. 16(a) clearly shows the specimen \#1, with a $9 \mathrm{~s}$ dwell, has several crack initiation points while

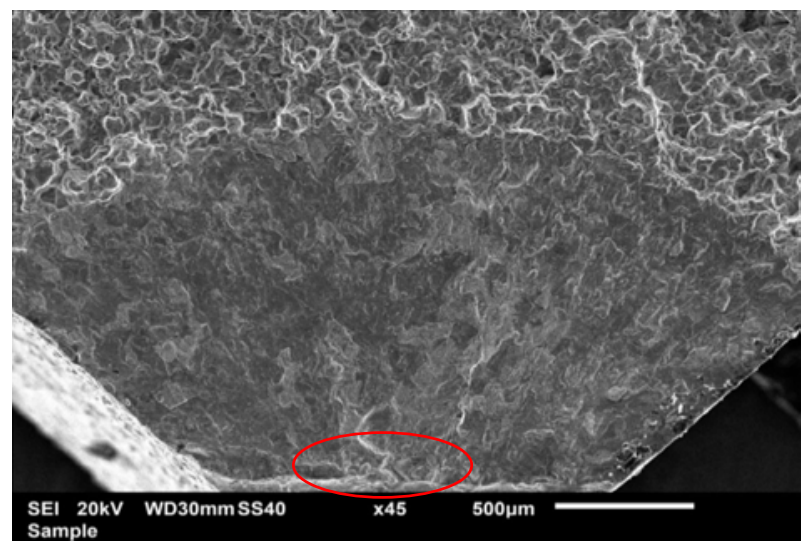

Fig. 20. Crack initiation of specimen \#6 at CCF loading (dwell = $108 \mathrm{~s}, n=10,000: 1$ ) Red oval indicates the crack initiation zone. (For interpretation of the references to colour in this figure legend, the reader is referred to the web version of this article.)
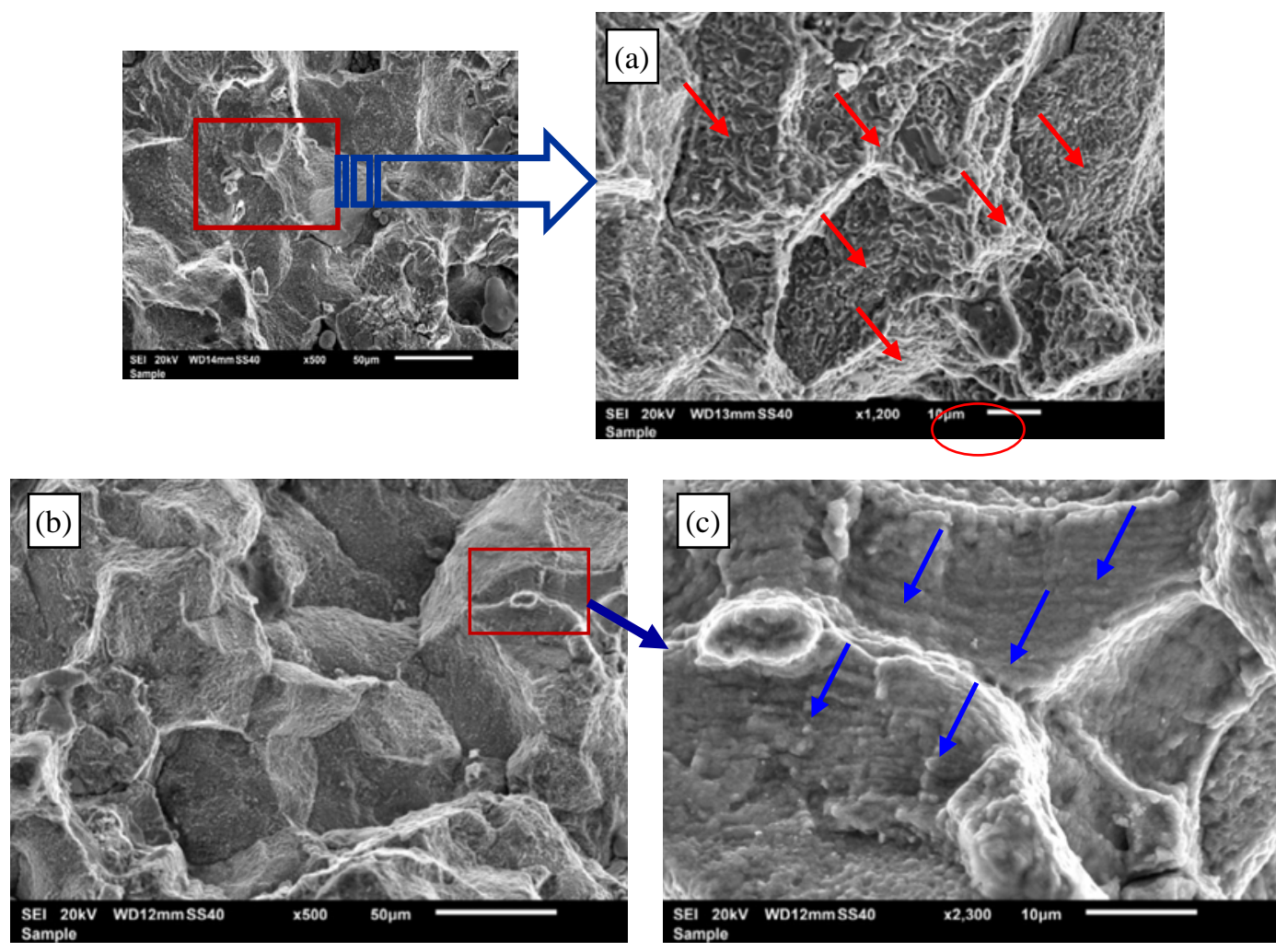

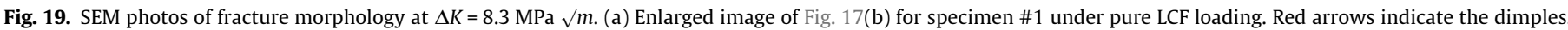

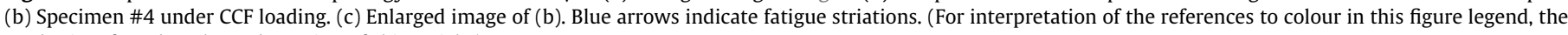
reader is referred to the web version of this article.) 


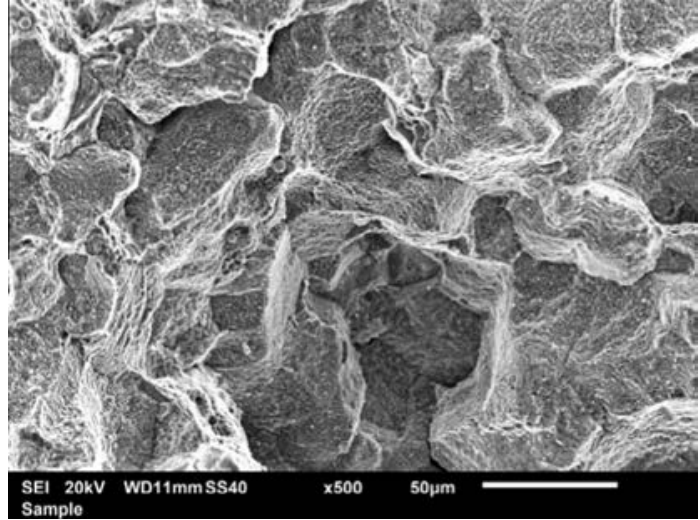

Fig. 21. Fracture morphology of specimen $\# 6, \Delta K=9.1 \mathrm{MPa} \sqrt{m}$.

specimen \#3, with long dwell time, has only one crack source as illustrated in Fig. 16(b). This situation consistent with the long dwell time at elevated temperature causes the superalloy GH2036 defect sensitivity. As noted in Fig. 17(a), a large amount of oxide forms on the crack face in addition to fatigue striations. Also of significance is that the crack surface is highly oxidized for the specimen at pure LCF loading with a long dwell time in Fig. 17(c).

At a higher $\Delta K$ of $8.3 \mathrm{MPa} \sqrt{m}$, the fracture surface of specimen \#1, with a $9 \mathrm{~s}$ dwell time, is a mixture of intergranular and transgranular failure (Fig. 17(b)). However, for the specimen \#3, with a long dwell time at a higher $\Delta K$, an intergranular fracture appears dominant (Fig. 17(d)). A change in the failure mode from transgranular fatigue crack propagation to intergranular fatigue propagation causes the stepwise increase in higher crack growth rates (see Fig. 8).

\subsubsection{The difference between pure LCF and CCF tests}

In the case of CCF loading with short dwell time, SEM observation shows that multiple crack initiation sites for specimen \#4 are at the surface (Fig. 18), similar to pure LCF loading (Fig. 16(a)). Fig. 19(a) shows the enlarged image of Fig. 17(b). In the high magnification SEM image, the fatigue crack growth of specimen \#1, under pure LCF loading at higher $\Delta K$, reveals dimples. In contrast, obvious fatigue striations are observed in the enlarged image of Fig. 19(b) for specimen \#4, under CCF loading at higher $\Delta K$, as shown in Fig. 19 (c). These fatigue striations induced by superimposed HCF loading are important for decreasing the fatigue crack growth rate.

\subsubsection{The effect of cycle ratio under CCF tests}

Fig. 20 depicts that the crack source for specimen \#6, under CCF loading with a cycle ratio of $10,000: 1$, is different from specimen \#4 with a cycle ratio of 1000:1 in Fig. 18. It is well known that superalloy GH2036 is defect sensitivity with a long dwell time at elevated temperature, which eventually leads to the observed only one crack initiation zone. At a higher $\Delta K$, the fracture surface of specimen \#6 shows intergranular failure (Fig. 21) instead of a mixed transgranular and intergranular failure for specimen \#4. Consequently, a stepwise increase in crack growth rate occurs at a higher $\Delta K$ under CCF loading with a long dwell time (see Fig. 12).

In addition, oxide thicknesses of the specimens \#4 and \#6 under CCF loading are compared as shown in Fig. 22. The maximum oxide thickness of specimen \#4 is $4.147 \mu \mathrm{m}$ (Fig. 22(a)), while the minimum and maximum values are $5.340 \mu \mathrm{m}$ and $15.780 \mu \mathrm{m}$ for specimen \#6, respectively (Fig. 22(b)). The oxidation thickness of specimen \#6 with a high cycle ratio $(n=10,000: 1)$ is considerably greater than that of specimen \#4, resulting in a faster crack growth rate.

\section{Conclusions}

Overall our studies establish an experimental system at elevated temperature with a new CCF loading scheme and a design of fixture to achieve a noninterfering treatment of the HCF and LCF loading by employing a load train assembly with three movable joints. Then pure LCF and CCF tests on specimens cut from an actual forged turbine disc of GH2036 superalloy were performed in laboratory air at $550{ }^{\circ} \mathrm{C}$. Compared with previous work, our results confirm that superimposed small high frequency HCF amplitudes delay the crack growth rate using LCF tests for GH2036 superalloy. In addition, as the cycle ratio increases under CCF loading, the fatigue crack growth rate accelerates. From SEM observations, all of the cracks initiate from the surface of the starter notches. A change in the failure mode from transgranular to intergranular causes the stepwise increase in fatigue crack growth rates under a long dwell time. Furthermore, the oxidation thickness of the specimen under CCF loading with a larger cycle ratio is significantly greater than a small cycle ratio. In future research, crack growth rate corresponding to the creep damage should be considered to investigate crack growth behavior at elevated temperature.
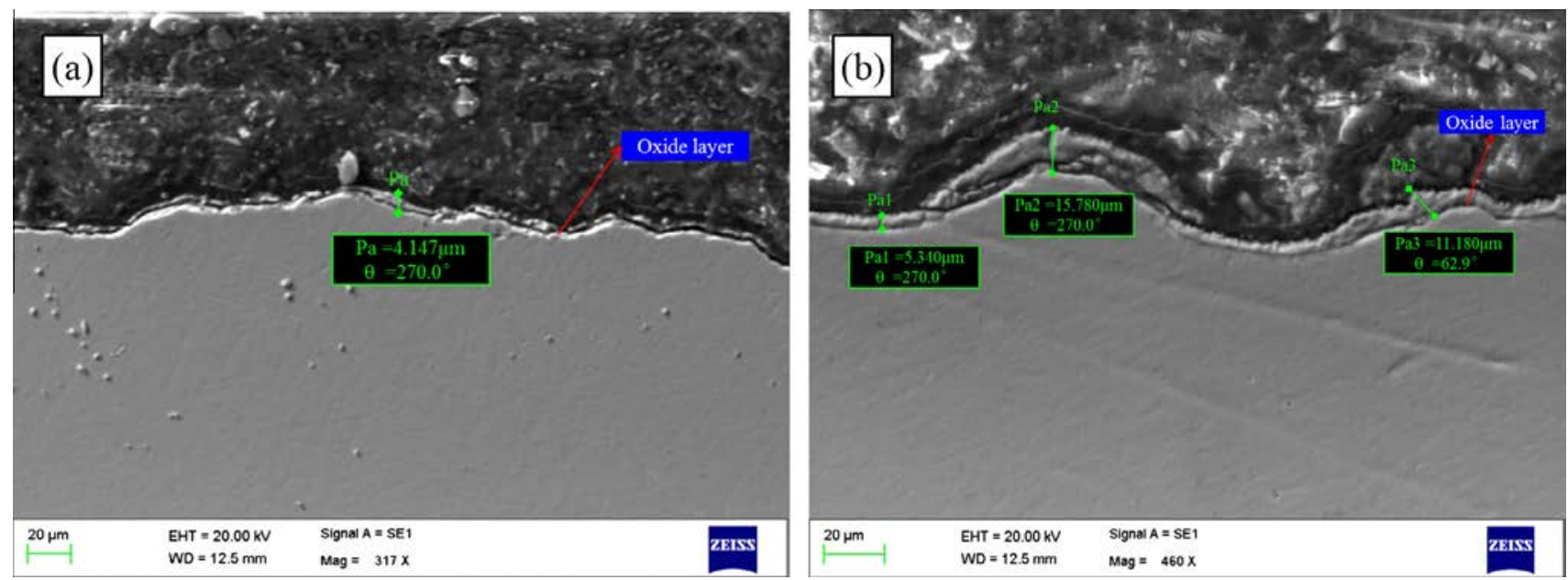

Fig. 22. Oxidation thickness. (a) Specimen \#4, and (b) specimen \#6. 


\section{Acknowledgements}

We greatly thank the financial support from National Natural Science Foundation of China (NSFC) $(51305012,51375031)$ and Aviation Science Fund of China (2014ZB51).

\section{References}

[1] Hu DY, Wang RQ. Combined fatigue experiments on full scale turbine components. Aircr Eng Aerosp Tec 2013;85(1):4-9.

[2] Wang RQ Cho CD, Nie JX. Combined fatigue life test and extrapolation of turbine disk mortise at elevated temperature. J Eng Gas Turb Power - Trans ASME 2005;127(4):863-8.

[3] Hou NX, Wen ZX, Yu QM, et al. Application of a combined high and low cycle fatigue life model on life prediction of SC blade. Int J Fatigue 2009;31 (4):616-9.

[4] Schweizer C, Seifert T, Nieweg B, et al. Mechanisms and modeling of fatigue crack growth under combined low and high cycle fatigue loading. Int J Fatigue 2011;33(2):194-202.

[5] Suresh S. Fatigue of materials. 2nd ed. Cambridge: Cambridge University Press; 1988.

[6] Hawkyard M, Powell BE, Hussey I, et al. Fatigue crack growth under the conjoint action of major and minor stress cycles. Fatigue Fract Eng Mater Struct $1996 ; 19(2 / 3): 217-27$.

[7] Hall RF, Powell BE. Crack growth in IMI829 at $550{ }^{\circ} \mathrm{C}$ under combined high and low cycle fatigue. Mat High Temp 2002;19(1):1-8.

[8] Powell BE, Hawkyard M, Grabowski L. The growth of cracks in Ti-6Al-4V plate under combined high and low cycle fatigue. Int J Fatigue 1997;19:167-76.

[9] Byrne J, Hall RF, Powell BE. Influence of LCF overloads on combined HCF/LCF crack growth. Int J Fatigue 2003;25:827-34.

[10] Ding J, Hall RF, Byrne J, et al. Fatigue crack growth from foreign object damage under combined low and high cycle loading, Part I: experimental studies. Int J Fatigue 2007;29:1339-49.
[11] Ouyang J, Wang Z, Song D, et al. Influence of high frequency vibrations on the low cycle fatigue behavior of a superalloy at elevated temperature. ASTM STP 942 Philadelphia; 1988. p. 961-71.

[12] Yan XJ, Nie JX. Fatigue of turbine blade. Beijing: Science Press; 2014.

[13] Dungey C, Bowen P. The effect of combined cycle fatigue upon the fatigue performance of TI-6AL-4V fan blade material. J Mater Process Tech 2004;153 (22):374-9.

[14] Fleck NA, Smith, RA. A discussion of mechanisms for accelerated and retarded fatigue crack growth. In: Proceeding of sixth international conference on fraction, Delhi, India; 1984.

[15] Editorial committee of handbook of Chinese aviation material handbook Chinese aviation material handbook. Beijing: China Standard Press; 2002.

[16] Xie ML, Zhong PD, Xi NS, et al. Analysis of fracture failure of fir-tree serrations of stage II turbine disks. Eng Fail Anal 2000;7:249-60.

[17] Wang RQ, Wei JM, Hu DY, et al. Investigation on experimental load spectrum for high and low cycle combined fatigue test. Propul Power Res 2013:2 (4):235-42.

[18] Xu XL, Yu ZW. Investigation of damage features of a turbo-disk. Eng Fail Ana 2009:16:1554-61.

[19] Weser S, Gampe U, Raddatz M, et al. Advanced experimental and analytical investigations on combined cycle fatigue (CCF) of conventional cast and single-crystal gas turbine blades. In: Proceeding of ASME turbo expo 2011, Vancouver, Canada; 2011.

[20] Mendia L, Estensoro FJ, Mary C, et al. Effect of combined cycle fatigue on Ti6242 fatigue strength. Proc Eng 2011;10:1809-14.

[21] Hu DY, Wei JM, Wang RQ, el al. An experimental test rig and measurement method for combined high and low cycle fatigue crack growth test at elevated temperature. Chinese Patent, Patent number: 201410853812.0; 2014.

[22] Hu DY, Wang RQ, Wei JM, el al. A test rig and methodology to measure the amplitude of HCF loading in a combined high and low cycle fatigue experiment. Chinese Patent, Patent number: ZL201310567800.7; 2015.

[23] Padula II SA, Shyam A, Ritche RO, et al. High frequency fatigue crack propagation behavior of a nickel-base turbine disk alloy. Int J Fatigue 1999;21:725-31.

[24] Pickard AC. The application of 3-dimensional finite element methods to fracture mechanics and fatigue life predictions. UK: Warley; 1986. 